\title{
Effectiveness of a tailored moisturizer on chemotherapy-induced skin dryness among breast cancer patients: Randomized controlled clinical trial
}

Danbee Kang, PhD1, Im-Ryung Kim AOCN, MSN ${ }^{2}$, Young Hyuck Im, MD PhD³, Yeon Hee Park, MD PhD ${ }^{3}$, Jeong Eon Lee, MD PhD Seok Jin Nam, MD PhD ${ }^{4}$, Hyeokgon Park, MSc ${ }^{5}$, Eunjoo Kim, PhD ${ }^{5}$, Haekwang Lee, PhD ${ }^{5}$, Eliseo Guallar MD, DrPH ${ }^{1,6}$, Jin Seok Ahn, MD PhD ${ }^{3}$, Juhee Cho MA, PhD 1,6

1Department of Clinical Research Design and Evaluation, SAIHST, ${ }^{2}$ Cancer Education Center, ${ }^{3}$ Division of Hematology/Oncology, Department of Medicine, 4 Department of Surgery, Samsung Medical Center, Sungkyunkwan University School of Medicine, Seoul, Korea

${ }^{5}$ Skin Research Institute, AMOREPACIFIC R\&D Center, Yongin, South Korea,

${ }^{6}$ Departments of Epidemiology, Johns Hopkins Bloomberg School of Public Health, Baltimore, Maryland, USA

\section{Background /Purpose}

- Up to $60 \%$ of breast cancer patients with chemotherapy (Ctx) had skin dryness due to decreased sebum contents.

- To evaluate effectiveness of a tailored moisturizer on skin dryness due to chemotherapy among breast cancer patients.

\section{Methods}

- Study Design: double-blind randomized controlled trial

- Population: breast cancer patients with dryness after 1cylce of Ctx

- Intervention: tailored and general moisturizer until 1 month after completion of Ctx

- Primary outcome: skin dryness

- Secondary outcome: dermatologic QoL, distress due to dryness

- Trial registration \# : KCT0001372

\section{Results}

Characteristics of study populations

- 188 patients participated in the study and 174 patients (tailored, $\mathrm{N}$ = 59, General, $N=56$; Control, $N=61$ ) completed the study.

- Mean age was 48.2 and patient were well balanced in the 3 groups.

- $27.8 \%$ of participants had severe dryness at baseline.

Patient-reported skin dryness

- Patients with the tailored (8.5\%) and general moisturizer (8.9\%) reported less skin dryness than patients with no product $(27.9 \%)$ after the intervention (Figure 2)

Sebum level

- Sebum contents in cheek of tailored group was relatively less decreased during trial compared to the no product and general group. (Figure 3)

Distress due to skin dryness

- Patients with the tailored moisturizer also reported lower levels of stress due to skin dryness (1.5 vs. $2.8 ; P=0.01$ ) (Figure 4)

Dermatologic QoL

- Better dermatologic QoL in the tailored group $(2.0$ vs. $4.8 ; P<0.001)$ than no product group. (Figure 5)
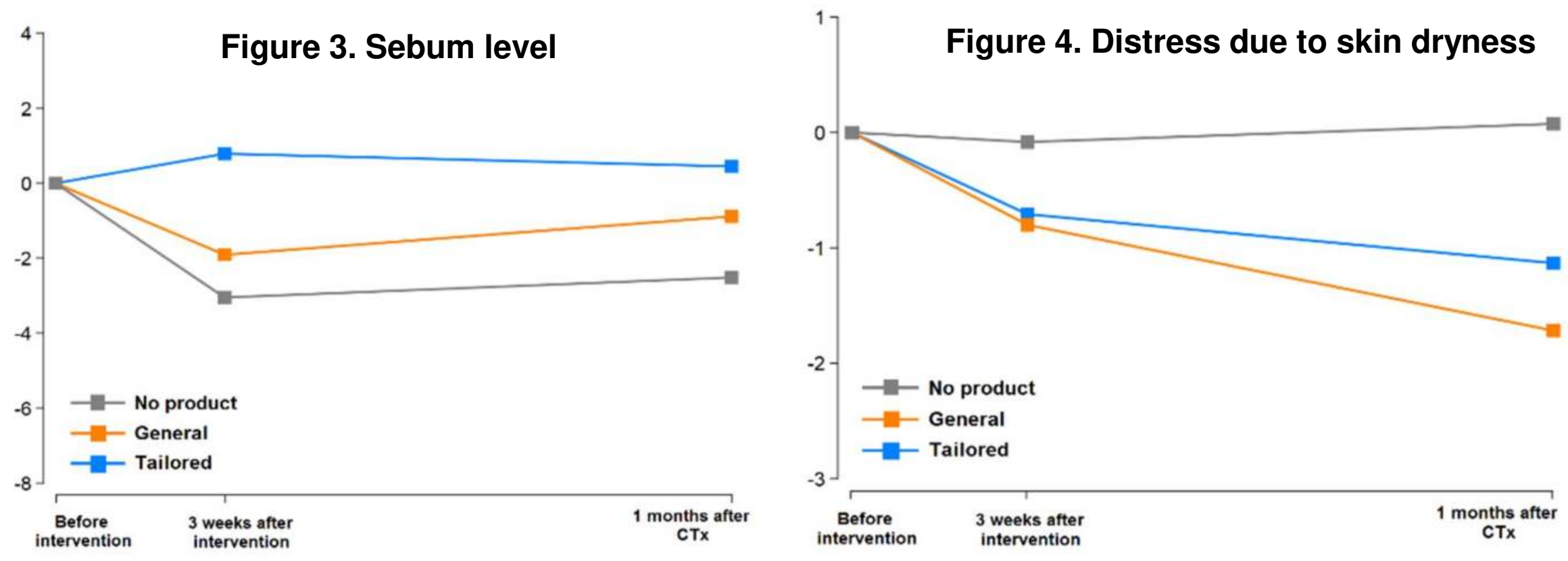

Conclusions

- Tailored moisturizer for chemotherapy-induced skin dryness was effective.

- Appropriate education and tailored intervention would be necessary to prevent skin dryness among breast cancer patients with chemotherapy.

\section{Acknowledgement}

